\title{
Finding Hidden Patterns in Complex Ventricular Ectopy
}

\author{
V Schulte-Frohlinde ${ }^{1,2}$, Y Ashkenazy ${ }^{1}$, PCh Ivanov $^{1,3}$, A Morley-Davies $^{4}$, L Glass $^{5}$, \\ AL Goldberger ${ }^{3}$, HE Stanley ${ }^{1}$ \\ ${ }^{1}$ Center for Polymer Studies and Department of Physics, Boston University, Boston, USA \\ ${ }^{2}$ Department of Physics, Harvard University, Cambridge, USA \\ ${ }^{3}$ Cardiovascular Division, Harvard Medical School, Beth Israel Deaconess Medical Center, \\ Boston, USA \\ ${ }^{4}$ Pilgrim Hospital, Boston, UK \\ ${ }^{5}$ McGill University, Montreal, Canada
}

\begin{abstract}
There has been much interest in analyzing the temporal organization of premature ventricular complexes. Different proposed mechanisms predict specific patterns. So far, these predictions have been mostly tested by inspecting short data strips. We present a novel method for representing cardiac time series as long as 24 hours using a temporal reorganization of the data. Our three dimensional rate-dependent histograms show the number of intervening beats or the interectopic time intervals for different sinus$R R$ intervals, an approach that may be useful to uncover hidden patterns of ectopy and to classify different dynamics of ectopy.
\end{abstract}

\section{Introduction}

Premature ventricular complexes (PVCs) are associated with an increased cardiac risk. Frequent PVCs are, for example, found in subjects with congestive heart failure. In the RR-time series they appear in complex patterns that often seem to be random (Figure 1). These patterns have been studied by determining statistical properties of long time series $[1,2]$, by analyzing an underlying mechanism by beat-to-beat inspection of short data-strips $[3,4,5]$, and by building theoretical models $[6,7,5,9,8]$. These studies have revealed a unexpected temporal structure in certain cases. We will introduce a presentation of 24-hour time series that allows us to visualize structure in the data and could provide a basis for classification of frequent PVCs.

\section{Data set}

We analyze a data set of RR-time series of 200 subjects with congestive heart failure. Out of these we have initially selected those subjects with the most frequent

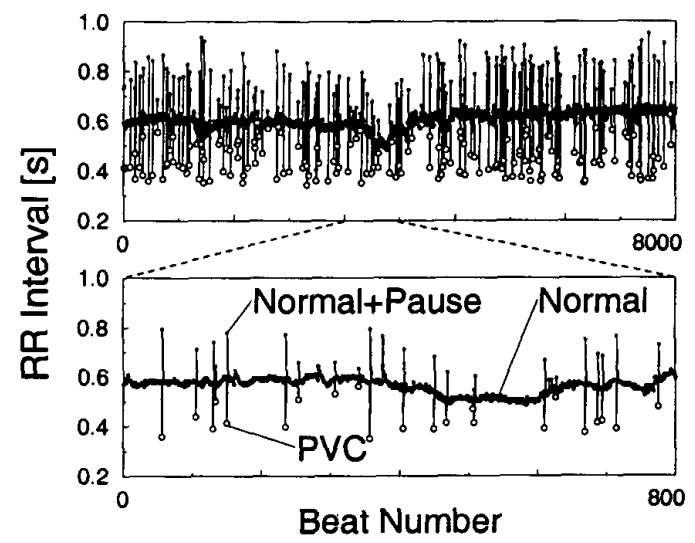

Figure 1. The RR-time intervals against beat number of a patient with congestive heart failure (subject 2 in the following figures). The upper panel shows 8000 beats, the lower panel is an enlargement of the upper figure containing only 800 beats. The downward spikes are the premature ventricular complexes; the upward spikes are normal beats after a compensatory pause. The distribution of the PVCs appears random.

PVCs (\#PVC>5000 in 24 hours). Here, we choose three subjects that show different patterns in their temporal PVC distribution. The sampling rate is $128 / s$.

\section{Conventional histograms}

At first, we construct a conventional histogram of the time intervals between consecutive PVCs. In Figure 2 we see broad and irregular peaks in the histograms of Subject 1 and 2. By contrast, subject 3 shows a broad distribution but no pronounced peaks.

A complementary presentation of the data is the 

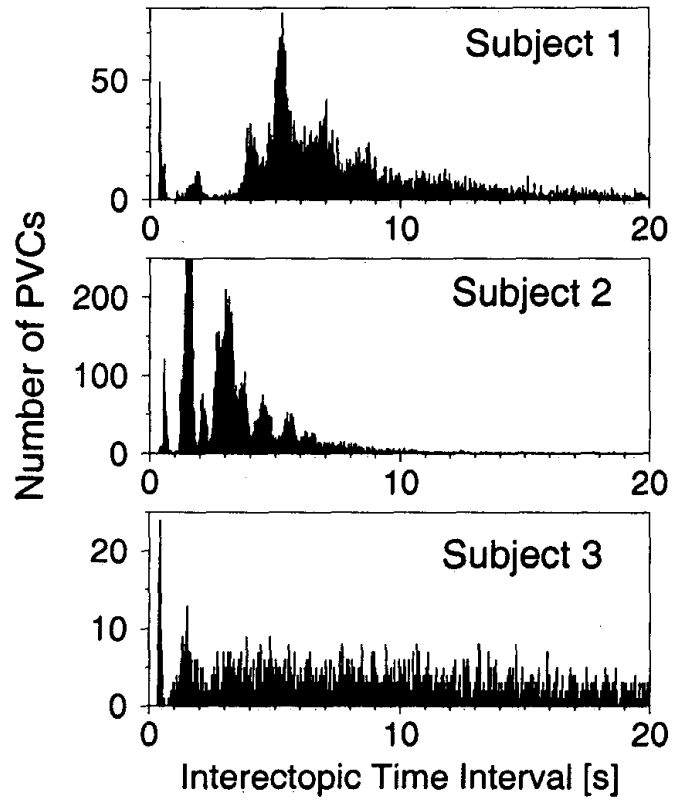

Figure 2. Histograms of the interectopic time intervals over 24 hours for three different subjects. For subject 1 and 2 the figure shows apparent peaks, whereas for subject 3 the histogram is smeared out.

histogram of the number of intervening sinus beats (NIBs) between two consecutive PVCs. The NIB-histograms of the three subjects are shown in Figure 3. Subject 1 displays peaks for even number $n \geq 4$ whereas subject 2 has most of the counts for $\mathrm{NIB}=1$. A broad distribution is observed in subject 3 .

\section{3D rate-dependent histograms}

To further investigate the different patterns found in the conventional histograms we study their dependence on the mean sinus beat intervals. We therefore add the sinus beat interval as a third dimension to the NIB-histograms.

These 3D-histograms are constructed in the following way. For each PVC in the time series, we count the NIB and the time interval until the next PVC. We determine the mean sinus beat interval in a window of 20 sinus beats centered around each PVC. Data are plotted with the mean sinus rate as the $x$-coordinate and the value for the NIB as the $y$-coordinate. The binning for the sinus beat period $T_{S}=$ $0.01 \mathrm{~s}$. The number of points is represented in a gray scale. Note that the definition of the gray scale is different for the three subjects as explained in Figure 4. The result is shown in Figure 5. Clear patterns emerge that were not evident

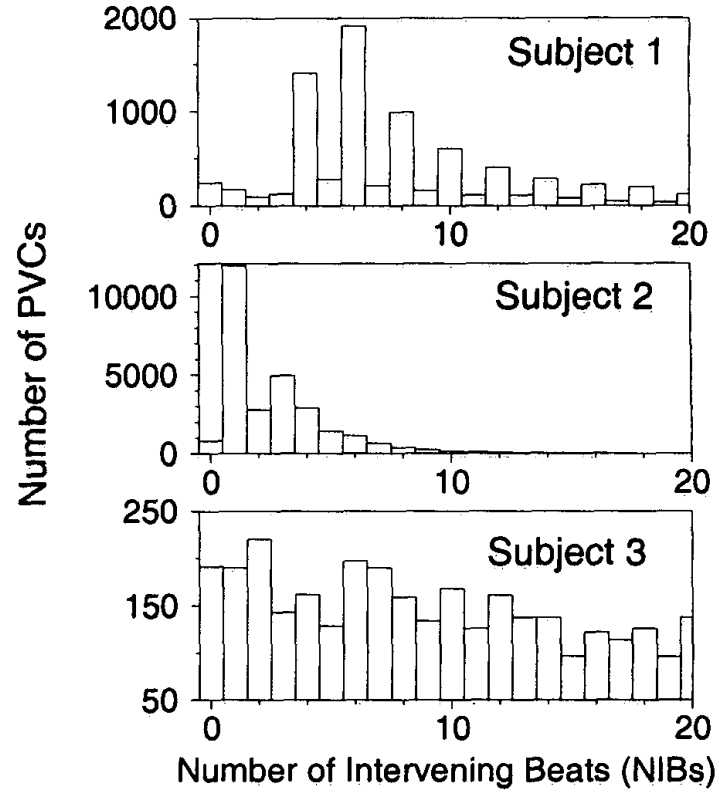

Figure 3. Histograms of the number of intervening sinus beats (NIB) over 24 hours for three different subjects. For subject one and two the figure shows a more pronounced structure, whereas for subject three the data are more "smeared out". None of the histograms shows only three peaks as predicted by the mathematical model of pure parasystole [9].

in previous figures. This structure differs from subject to subject. In subject 1 , the same NIB-structure is preserved over the whole $T_{S}$-range and is therefore also clearly seen in Figure 3. By contrast, in subject 2 we notice a change in the NIB-structure at $T_{S}=0.85 \mathrm{~s}$. For $T_{S}<0.85 \mathrm{~s}$ the most pronounced NIB-values are 1 and 3 , for $T_{S}>0.85 \mathrm{~s}$ we see more NIB-values at 2 and 4. Projecting this plot on its $y$-axes adds up the histograms for individual $T_{S}$ smearing out the peaks in Figure 3. The $T_{S}$-resolved NIB-histogram of subject 3 remains as unstructured as in Figure 3. The density of beats of this subjects is much lower than in the other two subjects due to the broader distribution.

We also determine the 3D-histograms for the interectopic time intervals (Figure 6) using the same binning for the sinus beat intervals as in Figure 5. A linear structure emerges in which all lines have a negative slope that becomes more negative with increasing interectopic time interval. The distance between the lines are different from subject to subject. 


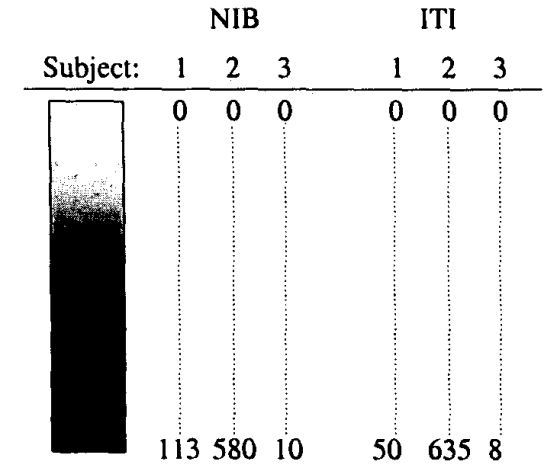

Figure 4. Definition of the gray scale for Figures 5 (NIB) and 6 (ITI=interectopic time interval). Black is associated with the maximum number of PVCs which differs from subject to subject. The values of these maxima are given in this figure.

\section{Discussion}

In this paper we have presented statistical methods to represent data of patients with frequent PVCs. Although from a clinical perspective all these patients might be considered similar since they all have more than 5000 PVCs $/ 24$ hours, the dynamics of the occurences of the PVCs is strikingly different in the three cases. We conjecture that this reflects different underlying physiological mechanisms.

One relatively uncommon physiological mechanism for frequent PVCs is pure parasystole. In pure parasystole the PVCs are generated from an independent ectopic pacemaker in the ventricles. In such a case the interectopic time intervals are integer multiples of the period $T_{V}$ of the ventricular pacemaker, leading to equidistant peaks in the histograms in Figure 2 and to equidistant lines with zero slope in Figure 6. For pure parasystole a mathematical model was developed that makes distinct predictions $[4$, $5,9,8]$. It is described by only two parameters: the ratio $T_{V} / T_{S}$ of the periods of the ventricular pacemaker $T_{S}$ and the sinus pacemaker $T_{S}$, and the ratio of the refractory time $\theta$ and $T_{S}$. The predictions of this model are formulated in terms of the NIBs. The parameter space spanned by $T_{V} / T_{S}$ on one axis and $\theta / T_{S}$ on the other axis is divided into regions. In each of these regions only three different values of NIBs are possible, such that for pure parasystole only three dark areas should be present in each vertical line in Figure 5. These predictions change when there is an interaction between the two pacemakers (modulated parasystole) [4]. The boundaries of the regions become blurred and some new regions appear.

Not surprisingly, the three cases analyzed here do not follow the predictions of pure parasystolic mechanism. One case of pure parasystole was found in the data set, and it will be discussed separately [10]. Other potential mechanisms for these arrhythmias are triggered activity and reentry [11]. However, theoretical models capable of predicting the rate dependence of the interectopic time interval and the NIBdistribution for these mechanisms have not been developed. This paper shows that patients with frequent PVCs may have different dynamical characteristics. Since different mechanisms may be associated with different risk, the classification of the dynamics of frequent PVCs merits further analysis.

\section{Conclusion}

The proposed 3D rate-dependent histograms may show hidden patterns in data records from 24-hour Holter recordings. They may be helpful in identifying complex mechanisms like variants of parasystole [10]. The resulting $3 \mathrm{D}$ rate-dependent histograms can be used for quantitative comparisons with theoretical models of arrhythmia.

\section{Acknowledgements}

This work was supported by the German Academic Exchange Service (DAAD), and by the NIH/NCRR Research Resource for Complex Physiological Signals(www.physionet.org).

\section{References}

[1] De Paola R, Wang HX, Norwood WI. Am J Physiol 1993;265:H1603. Wang HX, De Paola R, Norwood WI. Phys Rev Lett 1993;70:3671. Wang HX, De Paola R, Norwood WI. Phys Rev Lett; 1993;71:3039.

[2] Murakawa $Y$, Inoue $H$, Koide T, Nozaki A, Sugimoto T. Br Heart J 1992;68:589.

[3] Moe GK, Jalife JJ, Mueller WJ, Moe B. Circulation 1977;56:968.

[4] Courtemanche M, Glass L, Rosengarten MD, Goldberger AL, Am J Physiol 1989;257:H693.

[5] Courtemanche M, Glass L, Bélair J, Scagliotti D, Gordon D. Physica D 1989;40:299.

[6] Jalife JJ, Antzelevitch Ch, Moe GK. Pace 1982;5:911.

[7] Ikeda N, Yoshizawa S, Sato T. J theor Biol 1983; 103:439.

[8] Glass L, Goldberger AL, Bélair J. Am J Physiol 1986;251:H841.

[9] Glass L, Goldberger AL, Courtemanche M, Shrier A, Proc R Soc Lond A 1987;413:9.

[10] Schulte-Frohlinde V, Ashkenazy Y, Ivanov PCh, Glass L, Goldberger AL, Stanley HE. in preparation.

[11] Prystowsky EN, Klein GJ. Cardiac Arrhythmias. New York:McGraw-Hill, 1994. 
Subject 1

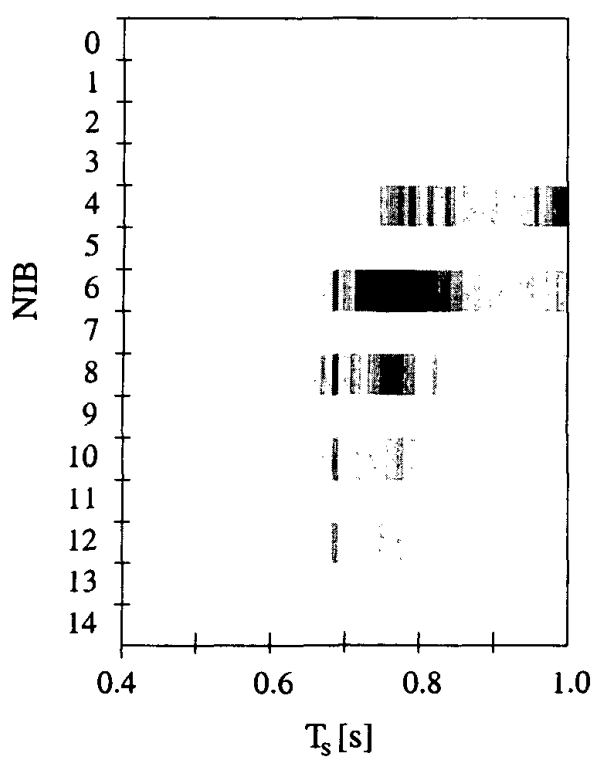

Subject 2

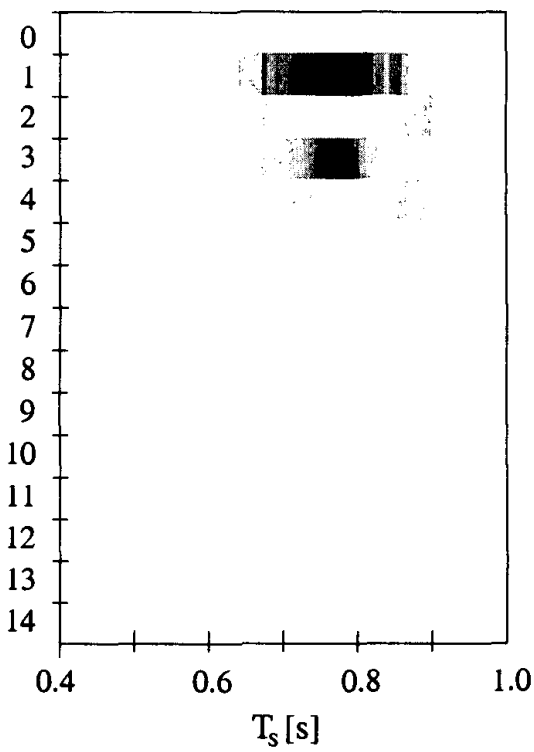

Subject 3

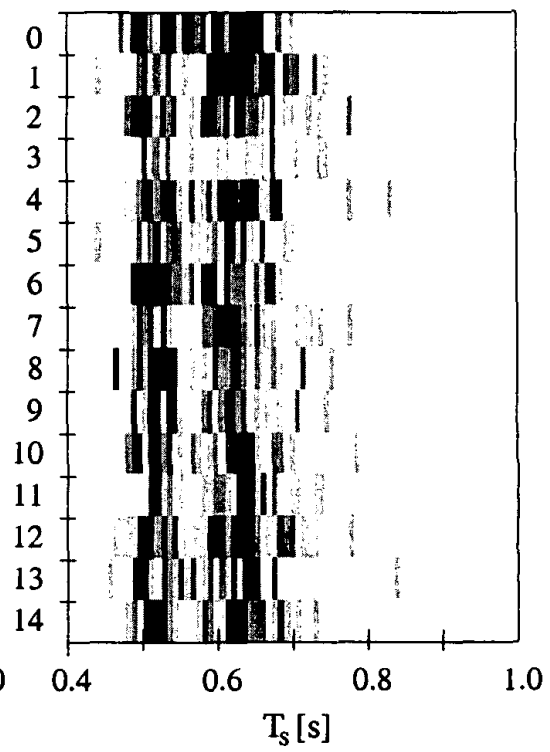

Figure 5. The 3D-histograms showing the number of intervening beats in dependence on the sinus beat interval $T_{S}$. For the gray scale see Figure 4 and text.

Subject 1

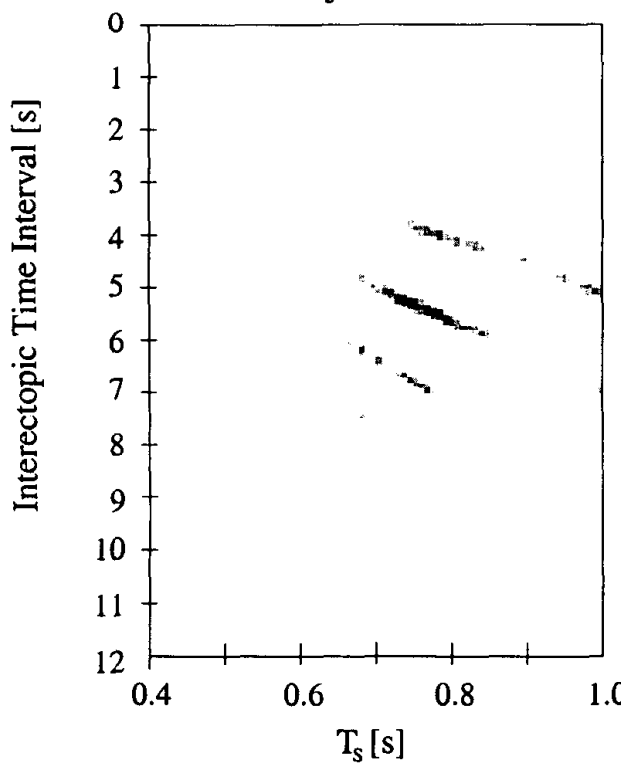

Subject 3

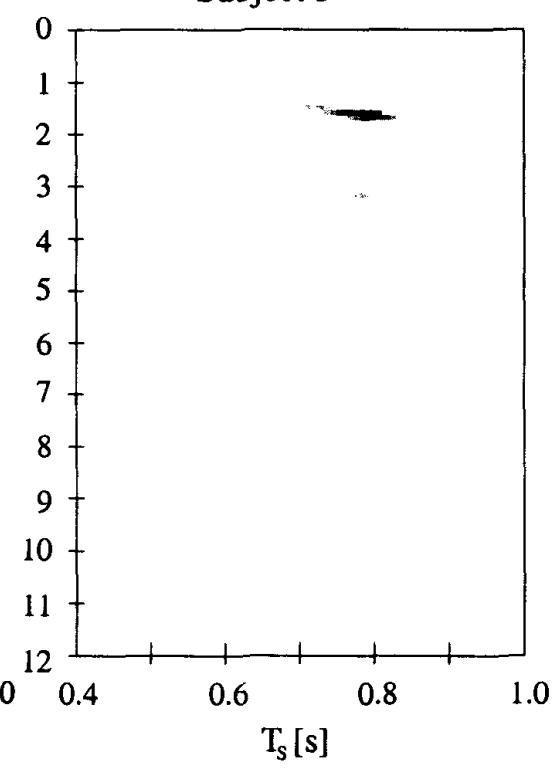

Subject 3

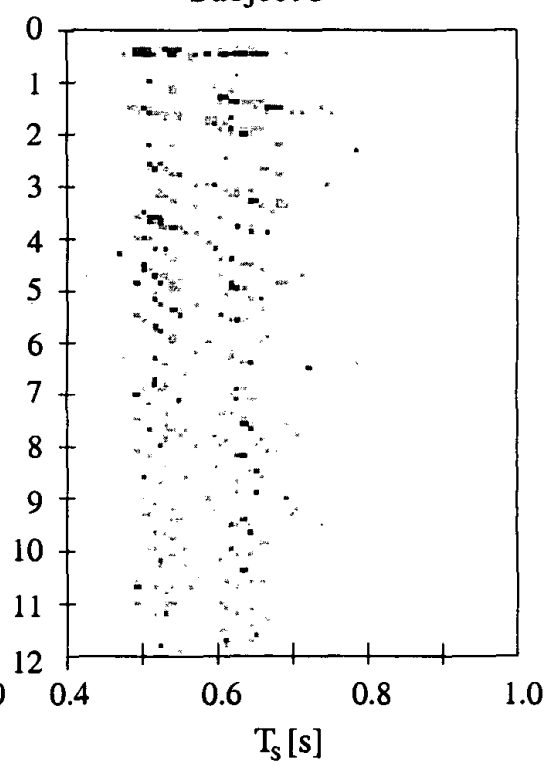

Figure 6. The 3D-histograms showing the interectopic time intervals in dependence on the sinus beat interval $T_{S}$. See text. 